\title{
Threonylcarbamoyladenosine tRNA Methylthiotransferase
}

National Cancer Institute

\section{Source}

National Cancer Institute. Threonylcarbamoyladenosine tRNA Methylthiotransferase. NCI Thesaurus. Code C131364.

Threonylcarbamoyladenosine tRNA methylthiotransferase (579 aa, $\sim 65 \mathrm{kDa}$ ) is encoded by the human CDKAL1 gene. This protein plays a role in the methylthiolation of transfer RNA. 